\title{
Visual acuity at two phases of the menstrual cycle
}

\author{
DENA SCHER \\ Marygrove College, Detroit, Michigan \\ DEAN G. PURCELL \\ Oakland University, Rochester, Michigan \\ and \\ SAM J. CAPUTO \\ Chicago School of Osteopathic Medicine, Chicago, Illinois
}

\begin{abstract}
Visual acuity, as accessed through letter identification, was investigated at two phases of the menstrual cycle (ovulation and menstruation). Reduced acuity was found at the time of ovulation. This finding is inconsistent with previous studies that have employed detection tasks and found increased sensitivity at ovulation. An analysis of the impact of the specific task on the visual system and the interaction of task with cycle phase provides a resolution to this apparent inconsistency.
\end{abstract}

Scher, Pionk, and Purcell (1981) demonstrated that dark-adapted visual sensitivity ( $\mathrm{d}_{\mathrm{e}}^{\prime}$ ) was increased at ovulation but was unchanged at other phases of the menstrual cycle. This finding was consistent with earlier work that had also found dark-adapted sensitivity to be increased at or around the time of ovulation (Barris, Dawson, \& Theiss, 1980; Ward, Stone, \& Sandman, 1978). In addition, Scher et al. (1981) found no effect of cycle phase under light-adapted conditions. There are two relatively simple ways in which to view the results of the above studies on the menstrual cycle. Kopell, Lunde, Clayton, and Moos (1969) argued for a general arousal hypothesis such that all visual performance would show an increase around the time of ovulation. The general arousal hypothesis was consistent with the idea that sensitivity increased at ovulation in order to increase a female's sensitivity to visual cues that might lead to copulation during this most fertile cycle phase. A second position that we would like to propose assumes that although the effects of ovulation may produce an increase in visual sensitivity, the effects of such an increase on visual performance may be task specific. That is, although visual sensitivity to a spot of light may increase at ovulation, in the dark-adapted eye, this does not mean that all conditions or tasks should show a similar result. Using the work of Weblin (1973) as a model, we would like to suggest that an increase in visual sensitivity at ovulation could simultaneously produce both an increase in the ability to detect a spot of light and a decrement in visual spatial acuity. Weblin (1973) demon-

Requests for reprints should be addressed to Dena Scher, Department of Psychology, Marygrove College, Detroit, MI 48221. strated in the mud puppy (Necturus) that, at any given adaptation level, the cones can respond across a range of three $\log$ units from threshold to saturation. Although stimuli that are at or just below the saturation level may produce maximum or near-maximum responses from the receptor, these stimuli are difficult to distinguish from one another because of the compression of response near the saturation point. In effect, the contrast or difference threshold is increased.

In applying Weblin's (1973) work to the effects of ovulation on visual perception, it is necessary to consider the following. The dark-adapted retina can easily be saturated by photopic stimuli that are approximately $3 \mathrm{log}$ units above threshold (Weblin, 1973). As saturation occurs, the image contrast is reduced until it reaches zero at $100 \%$ saturation. Saturation is most pronounced when the stimuli are presented in a brief flash of light to the dark-adapted retina. This is because adaptation is unlikely to occur to any great extent under such conditions. Most importantly, with stimuli that are near the point of producing saturation, any increase in sensitivity that increases the effectiveness of stimulation would only increase the degree to which saturation would take place and the degree to which contrast sensitivity would be reduced. Under conditions in which saturation took place, a dark letter presented on a light background might produce a visual response that would be at or near the receptor maximum. If the receptor was at or near its maximum response level, the light background of the letter would produce little or no increment in response above that produced by the letter itself. Consequently, the letter would be difficult or impossible to distinguish from the background and performance would suffer accordingly. In other words, although it 
would reduce the absolute threshold, an increase in sensitivity at ovulation would also increase the difference threshold and thus make discrimination of a letter from its background more difficult. The present experiment was designed to test the hypothesis that letter identification in the dark-adapted eye would be reduced at ovulation as a consequence of the visual system's increased sensitivity at that time (Barris et al., 1980; Scher et al., 1981).

In addition to the variable of primary interest (cycle phase), a secondary variable of target duration was tested. The latter variable was added to provide a parametric manipulation that would influence directly the degree of saturation of the visual system. The manipulation of target duration within the range of durations, and at the intensity employed in the present study, provides that all stimuli will fall within the critical duration for luminance summation (Stevens \& Hall, 1966). Accordingly, increases in duration produce an increase in the effective energy of the target. That is, a 20 -msec stimulus should produce more saturation and a greater reduction in target contrast than a 5-msec stimulus.

\section{METHOD}

\section{Subjects}

Data were collected on four 18-30-year-old female students. All subjects were paid, had normal visual acuity, and reported normal menstrual cycles of 26-29 days. These subjects had served in a previous series of experiments (Scher et al., 1981). They and the experimenter were not informed of the hypothesis being tested.

\section{Procedure}

Percent-correct forced-choice letter naming was collected at both the menstrual and the ovulatory phases of the menstrual cycle. Both basal body temperature and days from onset of menstruation were used to determine the exact phase of the menstrual cycle. Experimental data were collected on the 1st day of menses and on the day of the rise in a subject's basal body temperature. This would be the day after ovulation and was the day on which Barris et al. (1980) had found the greatest increase in visual sensitivity. To ensure accuracy of phase of testing, the basal body temperature was monitored throughout the menstrual cycle. The time course of the menstrual cycle for these subjects was well known, because their previous cycles had been monitored for other studies. Within subjects, the time of day of testing was held constant. Pretesting sessions of approximately 180 trials per day were run across 2 days to train the subjects and to select a target letter size that would yield approximately $75 \%$ correct with a 20 -msec target presentation.

During the experimental sessions, the observers were dark-adapted for $10 \mathrm{~min}$ prior to data collection. During a 1-h session, 60 trials were collected under each of the three target durations in random blocks of 10 trials per duration; each target letter was presented once, in random order, during a block. The subjects were required to respond on each trial even if they had to guess the identity of the presented letter. Just prior to each data-collection session, the subjects were given 10 practice trials under each of the three target durations.

\section{Apparatus}

An Iconix four-channel tachistoscope was used to provide fixation fields and to present stimuli. The target stimuli consisted of the 10 capital letters A, H, I, M, T, U, V, W, X, and Y. These letters were presented to a dark fixation field centered on a point $0.5^{\circ}$ to the right of a dim red fixation dot. The target letters were presented as black-onwhite stimuli and were front lighted. The white field in which they were presented was $3^{\circ}$ square. The luminance of this field was set at 75.38 $\mathrm{cd} / \mathrm{m}^{2}$. Three target durations-5, 10, and $20 \mathrm{msec}-$ were tested. In order to meet the pretesting criteria of $75 \%$ correct with a 20 -msec stimulus, the size of the stimulus letters was varied across observers. Accordingly, the visual angles subtended by the various stimulus sets ranged from $0.08^{\circ}$ to $0.12^{\circ}$ with proportions maintained. A subject viewed the stimuli with her eye of greatest acuity.

\section{RESULTS}

A repeated-measures analysis of variance was performed on the mean percent-correct target report for each of the target durations for each of the two phases of the menstrual cycle. The main effect of cycle phase was significant $[F(1,3)=13.99, p<.05]$, with subjects performing better at the onset of menstruation than on the day after ovulation. The effect of target duration was also significant $[\mathrm{F}(2,6)=14.20, \mathrm{p}<.01]$, with target report being reduced at the longer target durations. There was no significant interaction between these two variables. $[F(2,6)=1.31]$. The results of the experiment are displayed in Figure 1.

\section{DISCUSSION}

It is important to note that, considered alone, the present experiment does not allow a determination of whether reduced performance at ovulation or enhanced performance at menstruation was demonstrated. Such a determination would have required that other phases be tested to establish baseline performance. In previous research in which we had established such a baseline, only ovulation was found to deviate from the other cycle phases (Scher et al., 1981). In light of this finding, it is possible to interpret the present result as being consistent with the hypothesis that an increase in visual sensitivity is associated with the ovulatory phase of the human female menstrual cycle. Assuming that ovulation also in-

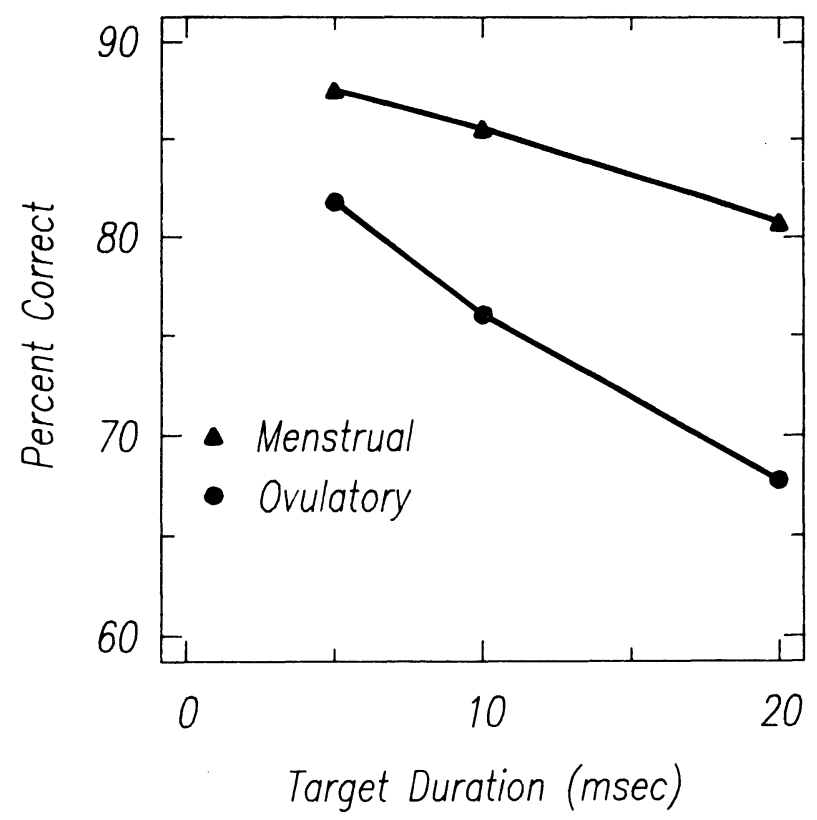

Figure 1. Percent-correct forced-choice letter identification as a function of target duration at the menstrual and ovulatory cycle phases. 
creased sensitivity in this study, we are left with the seemingly paradoxical finding that increased visual sensitivity can hamper target identification. However, the effect can be explained if one considers that, with increased sensitivity, there is a greater potential for the visual system to saturate at ovulation and thus reduce the contrast of any given stimulus. Any reduction in contrast of our already difficult-to-identify stimuli would have the effect of reducing performance even further. Evidence that our stimuli were within the luminance or energy range at which saturation could take place can be found in the effect that increasing letter duration had on performance. Increases in letter duration, and hence in the energy of the presentation, the physical counterpart of increased visual sensitivity, served to reduce target recognition. This affords a direct comparison of what occurs when target energy is directly manipulated and what occurs when analogous effects are produced as a consequence of increases in the sensitivity of the visual system. Both result in an increase in saturation and, consequently, in a reduction in performance on a letter-identification task.

It is unlikely that the present findings are attributable to motivational effects (see Parlee, 1973). The fact that Scher et al. (1981) found increased performance at ovulation and that the present study found decreased performance at ovulation suggests that it is even more unlikely that these results are attributable to nonperceptual mechanisms such as mood or motivation. If the present results and those of Scher et al. (1981) are attributable to mood change or motivation, then they should produce the same pattern with regard to ovulation and menstruation.

Although the results of the present study do not directly contradict the general arousal theory of the effects of ovulation on behavior (Kopell et al., 1969), they do suggest that care must be taken in predicting the direction in which arousal will drive performance. Furthermore, if the present data are attributable to increased sensitivity at ovulation, which in turn produces a greater tendency for cones to saturate, then these findings are more consistent with a retinal than with a central mechanism's producing the effect. The present study indicates that care must be taken in comparing results of studies that have investigated the effects of the menstrual cycle on perception but have used different tasks and different adaptation levels.

\section{REFERENCES}

Barris, M. C., Dawson, W. W., \& Theiss, C. L. (1980). The visual sensitivity of women during the menstrual cycle. Documenta Ophthalmologica, 49, 293-301.

Kopell, B. S., Lunde, D. T., Clayton, R. B., \& Moos, R. H. (1969). Variations in some measures of arousal during the menstrual cycle. Journal of Nervous and Mental Disease, 148, 180-187.

Parlee, M. B. (1973). The premenstrual syndrome. Psychological Bulletin, 80, 454-465.

Scher, D., Pionk, M., \& PURCell, D. G. (1981). Visual sensitivity fluctuations during the menstrual cycle under dark and light adaptation. Bulletin of the Psychonomic Society, 18, 159-160.

Stevens, J. C., \& Hall, J. W. (1966). Brightness and loudness as functions of stimulus duration. Perception \& Psychophysics, 1, 319-327.

Ward, M. M., Stone, S. C., \& Sandman, C. A. (1978). Visual perception in women during the menstrual cycle. Physiology \& Behavior, 20, 239-243.

Weblin, F. S. (1973). The control of sensitivity in the retina. Scientific American, 228(1), 71-79.

(Manuscript received for publication November 19, 1984.) 